\title{
Interface Fracture Models of Concrete Externally Reinforced by FRP Plates
}

\author{
Lei ZHANG ${ }^{1, a,{ }^{*}, \text { Ping-Hu LIU }}{ }^{2, b}$, Xiao-Peng GUO ${ }^{2, c}$, Yong YANG ${ }^{1, d}$, \\ Jun ZHENG ${ }^{1, e}$ \\ ${ }^{1}$ Yellow River Institute of Hydraulic Research, YRCC, Zhengzhou 450003, P.R. China \\ ${ }^{2}$ Yellow River Wanjiazhai Water Multi-purpose Dam Project Co., LTD, Pianguan 036412, P.R. China \\ ahkyzhanglei@163.com, bwjzlph@163.com, cguoxiaopeng2007@sina.com, \\ dyangyong@hky.yrcc.gov.cn, ${ }^{\mathrm{e}} 173728100 @ q q . c o m$ \\ ${ }^{*}$ Corresponding author
}

Keywords: Rigid Joint Model, Semi-Rigid Joint Model, Flexible Joint Model, Energy Release Rate, Interface Fracture Models Component.

\begin{abstract}
In this paper, the fracture models on FRP-concrete bonded interface are summarized and compared for concrete structures externally reinforced with FRP plates. The energy release rates of the unified model of bi-material interface fracture specimens under the transverse and moment loads by the rigid, semi-rigid, and flexible joint models are compared. The results show that the classical rigid joint model under-evaluates the compliance, and it thus predicts the approximate energy release rate of the specimen. The semi-rigid joint model also under-evaluates the compliance and energy release rate of the specimen. The flexible joint model introduces two interface compliance coefficients to describe the crack tip deformation under the shear stress and normal stress, and it thus improves the calculation accuracy of both the compliance and energy release rate.
\end{abstract}

\section{Introduction}

As a new kind of reinforcement material, FRP has been widely applied in reinforce and repair of concrete structures, depending on its excellent performance such as lightweight, high strength, corrosion resistance, low magnetic induction and low coefficient of thermal expansion. When FRP materials were used for reinforcing concrete structures, FRP sheet (fiber cloth and FRP plate) were attached to the surface of the concrete elements via high-strength adhesive, thus playing the role of reinforcement. FRP has become a highly focused subject in research \& development and application of water conservancy projects. Some scholars [1-6] studied the main failure mode of FRP in concrete bending resistance and shear reinforcement. It is shown from the results the bonding interface between FRP sheet and concrete was a critical part for concrete structure reinforcement by FRP sheet, but a weak position in structure. Tiny cracks might cause obvious cracks gradually and further result in structural damage. Therefore, the analysis on fracture mechanism and failure mode of FRP-concrete bonding interface has been a research focus in FRP-reinforced concrete structure in water conservancy projects.

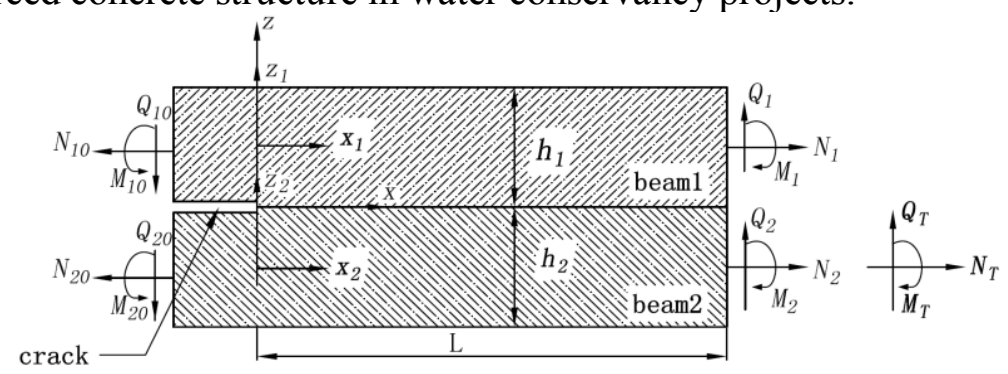

Fig. 1 The Bi-material Beam Model under Crack

In 1961, Kaplan [7] firstly applied the concept of fracture mechanics in the concrete, and performed fracture toughness test, thus causing great concern in academia at the time. Fracture model of bi-material 
beams was generally composed of cracking and non-cracking parts, and both parts were joined to form a node at the crack tip, as shown in Figure 1. Different interface joint model could be obtained after different hierarchical beam theories were applied, namely interface joint model based on deformation of the crack tip.

As shown in Figure 1, the interface crack tip was applied axial force, bending moment and lateral shear force, and the energy release rate and phase angle could be indicated with the internal forces.

\section{FRP-concrete Interface Fracture Model}

\section{Rigid Joint Model}

Williams [8] adopted the classical beam theory and concluded a simple expression of energy release rate for part of the force. Suo et al [9] made studies on interfacial crack problem between two isotropic elastic materials, and proposed the analytic formula of stress intensity factor based on the classical beam theory. Schapery et al [10] calculated the strain energy release rate of bi-material interface crack with classic beam / plate theory, and such the method was called as crack tip feature selection load as the load parameters, where characteristic load of crack tip was used as load parameter, and such the method was called the crack tip element analysis method. Davidson [11] promoted the concept of the crack tip, and analyzed the bi-material elastic plates with non-oscillatory or oscillatory singularity. Joint model based on classical beam theory was rigid joint model, which was a model used frequently in the literature, where cross sections of deformed non-cracked sub-laminates were assumed to remain in the same plane at a node, and to be still perpendicular to the neutral plane, by ignoring the relative axial displacement and the relative angle of each sub-laminate of the crack tip, without considering the local deformation of the crack tip, thus to form rigid nodes, as shown in Figure 2.

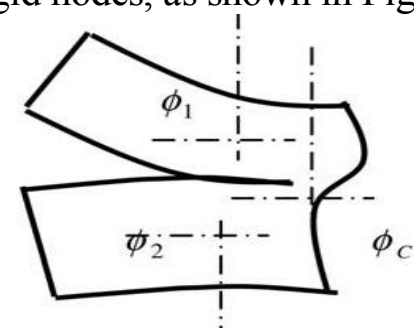

(a)The interface crack tip element

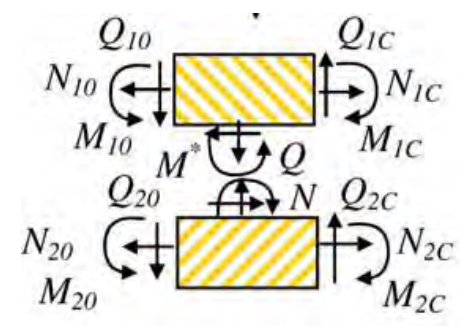

(b)The interface crack tip force

Fig. 2 Rigid Joint Fracture Model

To satisfy the equilibrium conditions, the model required three concentrated forces that did not actually exist at the crack tip, as shown in 2 (b), leading to great difference between the crack tip local stress distribution and the actual situation. Rigid joint model could not be used for analyzing local deformation of crack tip under load, but could only be used for analysis irrelevant to or less affected by node local deformation.

\section{Semi-Rigid Joint Model}

Although rigid joint model is simple and widely used, in the classical beam \& plate theory, shear deformation of sub-laminate in cracked and non-cracked FRP reinforced concrete beams was not considered, except for approximate analysis on bi-material interface [12, 13]. Li et al [14] adopted finite element method to conclude shear-related energy release rate formula: as for isotropic glass / epoxy specimens at a slenderness ratio of 8.33 , the energy release rate result was $13.7 \%$ more than the result as obtained with crack tip element analysis method (CTE). Bruno et al [15] studied influence of shear deformation on the energy release rate, they considered uncracked part of laminates as double Reissner-Mindlin plates as bonded via linear elastic interface, and introduced the interface model to ensure continuity of interface displacement of laminates, further concluded energy release rate analytical formula based on shear deformation factors, however, which was obtained by means of numerical computation. Thus, as for notched double-layer beams, the calculation and prediction of energy release rate should be based on double sub-laminate model that considers shear deformation, which made 
separate analysis on each sub-laminate, rather than considering uncracked beams as a whole $[9,10]$. Sub-laminate could be deformed separately and didn't follow plane cross-section assumption as shown in Figure 3.

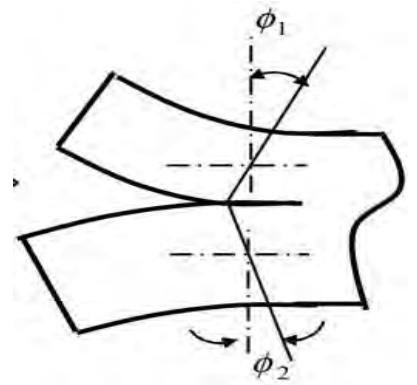

(a)The interface crack tip element

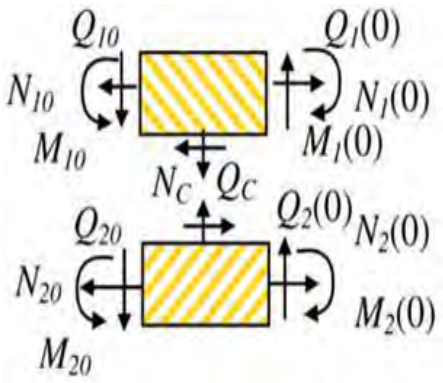

(b)The interface crack tip force

Fig. 3 Semi-rigid Joint Fracture Model

On basis of first-order shear deformation theory, Wang and Qiao [16] solved the energy release rate and the stress intensity factor of double-layer plate crack under normal loads, and worked out the analytical solution, further proposed shear deformation bi-material beam model. In this model the constraints of neutron corner in rigid joint model were removed and each sub-laminate had independent corner, but it was still assumed that the interfacial stresses had no impact on the displacement of each sub laminate, and thus shear deformation bi-material beam model could only consider local deformation of the crack tip, as called semi-rigid joint model. Crack tip node was deformable, and it was more suitable for fracture analysis on general cracked bi-material beam / plate.

In the semi-rigid joint model, due to the removal of constraints from the opposite corner of each sub-laminate, the crack tip required two concentrated force that didn't actually exist to satisfy the equilibrium conditions of crack tip elements, thus to ensure a certain difference between crack tip local stress distribution and actual situation. Therefore, the model was still underestimated in crack specimen flexibility and strain energy release rate.

\section{Flexible Joint Model}

In order to more accurately capture the local deformation of crack tip, Qiao and Wang [17] proposed a "bi-material interface deformable beam model." In the model deformation arising from each sub-laminate interface stress was further considered, interface-cracked bi- material beam was regarded as two intact and separate shear deformation sub-laminate, thus further introducing two interfaces softness parameters to describe shear deformation of two sub-laminate interfaces under the shear stress and normal stress, thus simulating the crack tip local deformation, resulting in the analytical solution of the crack tip deformation; it was called as flexible joint model, shown in Figure 4. Since each sub-laminate could be freely deformed, in flexible joint model, the crack tip satisfied its own equilibrium conditions.

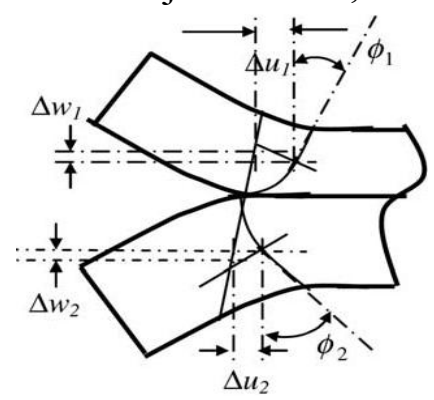

(a)The interface crack tip element

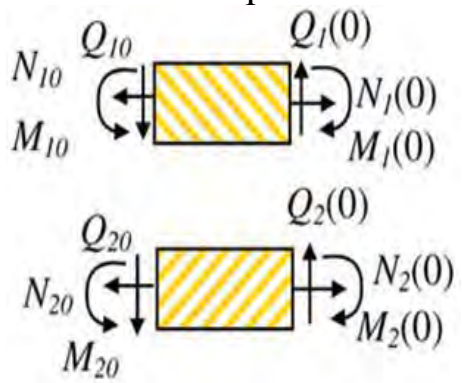

(b)The interface crack tip force

Fig. 4 Flexible Joint Fracture Model

Compared with rigid joint model in traditional composite beam / plate theory $[9,10]$, as well as semi-rigid model $[18,19]$ in two sub-laminate shear beam / plate theory, In the flexible joint model, the effect of interfacial deformation on the bi-material beam was considered, thus improved calculation accuracy obviously. 


\section{Comparison of Different Interface Joint Model}

In order to compare and analyze the different joint models, as shown in Figure 5, the bi-material interface fracture specimen was taken as the research object. Figure 5 (a) showed a mixed-type interfacial fracture specimen under vertical loads, and Figure 5 (b) showed a mixed-type interfacial fracture specimen under equivalent bending moment. Rigid joint model, semi-rigid joint model and flexible joint model as well as numerical analysis were respectively adopted to compare the orthogonalized energy release rate of different fracture specimen as crack propagation as shown in Figure 5. Assuming that all the sub-laminate o fracture specimens were made of isotropic material, the width of each specimen was equal to unit width, the numerical analysis solution is as exact solution.

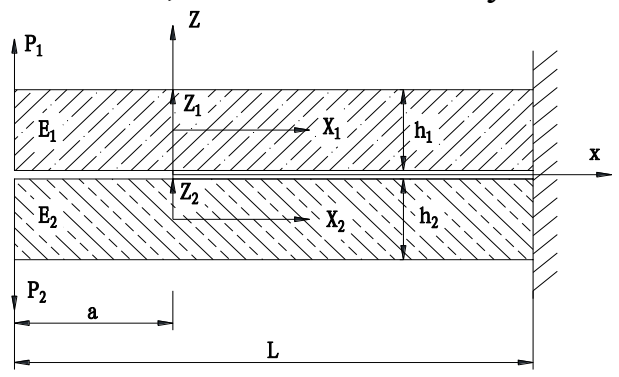

(a) Specimen under the vertical load

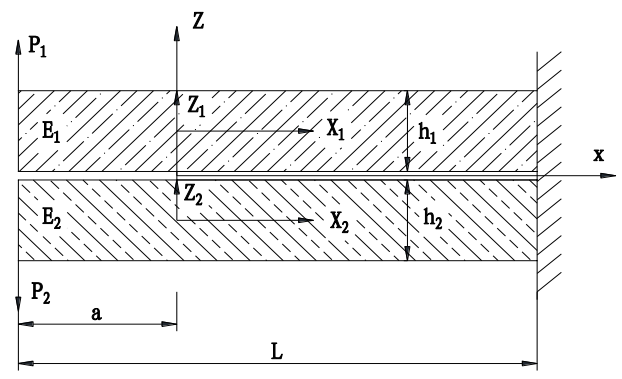

(b) Specimen under the equivalent bending moment

Fig. 5 The Unified Bi-material Interface Fracture Specimens

\section{Energy Release Rate of Interface Fracture Specimens under Vertical Load}

Figure 6 showed comparison of orthogonalized energy release rate of same bi-material fracture specimen under vertical loads as crack propagation respectively basing on rigid joint model, semi-rigid joint model and flexible joint model as well as numerical analysis.

The results showed that: (1) When the crack slenderness ratio was larger (crack length / sub-laminate thickness ratio), the four models' energy release rate would tend to the same constant; (2) When the crack slenderness ratio was smaller, rigid joint model was obviously different with the other three models in predicting the energy release rate, indicating that the rigid joint model could not accurately describe the interfacial fracture characteristics of interface fracture specimens under vertical loads; (3) compared with Finite Element Analysis' solution, in the solution of semi-rigid joint model the energy release rate of interfacial fracture specimens was underestimated, because semi-rigid joint model only considered the relative rotation of each sub-laminate among all the local deformation of the crack tip; (4) in flexible joint model all local deformation of the crack tip was considered, and its results was close to the solution of numerical analysis, in addition that crack slenderness ratio was close to 0 , the error between the two solutions was less than $1.5 \%$.

The above content indicated local deformation of crack tip made greater impact on accurate judgment to interfacial fracture specimen's energy release rate.

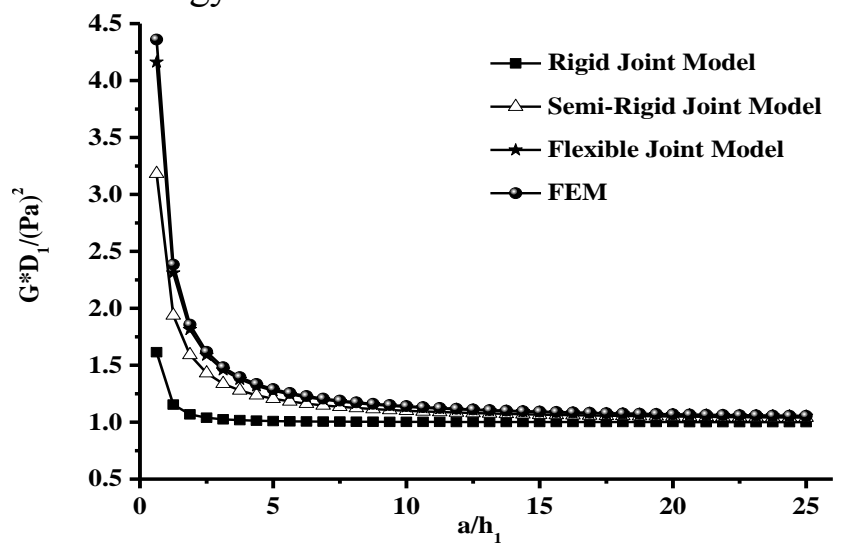

Fig. 6 Energy Release Rate by Different Interface Fracture Model under Vertical Load 


\section{Energy Release Rate of Interface Fracture Specimens under Equivalent Bending Moment}

Figure 7 showed comparison of orthogonalized energy release rate of same bi-material fracture specimen under bending moment loads (Fig. 5 (b)) as crack propagation respectively basing on rigid joint model, semi-rigid joint model and flexible joint model as well as numerical analysis.

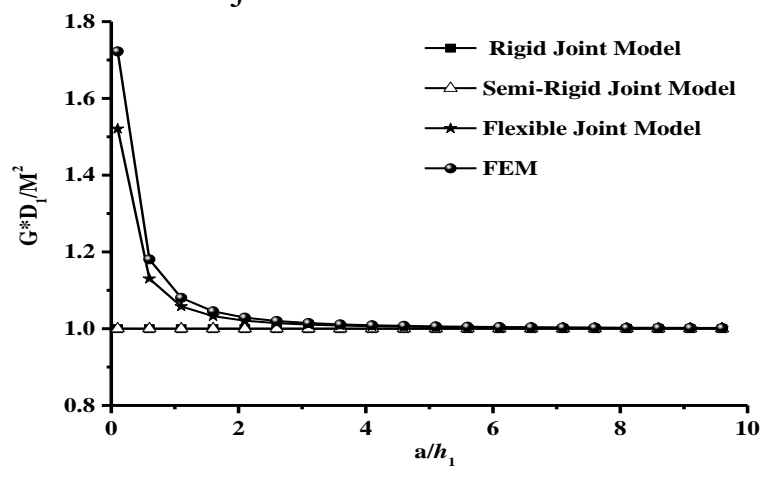

Fig. 7 Energy Release Rate by Different Interface Fracture Model under Equivalent Bending Moment

The results showed that: (1) under the equivalent bending moment, there was no shear force in each sub-laminate. Due to geometric symmetry of sub-laminate material of unified interface fracture specimens, under the equivalent bending moment, the relative corner of local deformation of the crack tip was a constant. Therefore, under the equivalent moment load, as for any geometrically symmetric interface crack specimen, energy release rate was exactly the same under rigid joint model and semi-rigid joint model; (2) energy release rate as predicted with semi-rigid joint model was a fixed constant; (3)the energy release rate of flexible joint model and numerical analysis sharply decreased as the crack slenderness ratio increased, and finally tended to be a specific constant.

Although the energy release rate with crack slenderness ratio was not same under these four models predict, but when slenderness ratio $a / h_{1}$ was greater than 6:00, the energy release rates under all three models tended to be specific constants. In actual tests, the crack slenderness ratio often ranged from 5 to 15 , indicating that under the equivalent moment load, as for any geometrically symmetric interface crack specimen, semi-rigid joint model could give a more accurate solution, this also showed that the rigid and semi-rigid joint models were capable to accurately predict the fracture characteristics of interface crack specimen containing symmetric sub-laminate under equivalent moment load.

\section{Summary}

The paper reviewed and compared FRP- concrete interface fracture mechanics models. Rigid joint model, semi-rigid joint model and flexible joint model as well as numerical analysis were respectively adopted to compare the orthogonalized energy release rate of unified bi-material fracture specimen as crack propagation under vertical loads and equivalent bending moment.(1) in the classical rigid model, assuming that two uncracked sub-laminates were under same deformation after bearing load, local deformation of the crack tip was ignored, the two sub-laminate interface thickness was zero, therefore, in the model the flexibility of the specimen was underestimated, and thus strain energy release rate's approximate solution was only obtained;(2) in semi-rigid joint model the constraints of opposite corner were removed, two sub-laminates had separate corner, but it was still assumed fracture interface wasn't deformed under the interfacial shear stress and normal stress. Such the assumption was deviated from the actual situation, and thus underestimated the local deformation and strain energy release rate of the crack tip;(3) in flexible joint model, two interface flexibility parameters were adopted to describe the shear deformation of two sub-laminate interface under shear stress and normal stress, thus to effectively describe local deformation of the crack tip, thus greatly improving the accuracy of fracture parameters.

\section{Acknowledgement}

This work was financially supported by the National Natural Science Foundation of China (51209102), 
and fundamental research funds for the central nonprofit research institutions (HKY-JBYW-2013-04).

\section{References}

[1]Chen J F, Teng J G, Shear capacity of FRP-strengthened RC beams: FRP debonding, Constr. Build. Mater., 17(1) (2003) 27-41.

[2]Tumialan G, Belarbi A, Nanni A, et al, Reinforced concrete beams strengthened with CFRP composites: failure due to concrete cover delamination, Center for Infrastructure Engineering Studies, University of Missouri, Rolla, 1999.

[3]Garden H N, Hollaway L C, An experimental study of the failure modes of reinforced concrete beams strengthened with prestressed carbon composite plates, Compos. Part B-Eng., 29(4) (1998) 411-424.

[4]Bonacci J F, Maalej M, Externally bonded fiber-reinforced polymer for rehabilitation of corrosion damaged concrete beams, Aci. Struct. J., 97 (5) (2000) 703-711.

[5]Al Sulaimani G J, Sharif A, Basunbul I A, et al, Shear repair for reinforced concrete by fiberglass plate bonding, Aci. Struct. J., 91(4) (1994) 458- 464.

[6]Chajes M J, Januszka T F, Mertz D R, et al, Shear strengthening of reinforced concrete beams using externally applied composite fabrics, Aci. Struct. J., 92(3) (1995) 295-303.

[7]Kaplan M F, Crack propagation and the fracture of concrete, Journal of the American Concrete Institute, 58(5) (1961) 591-610.

[8]Williams J G, On the calculation of energy release rates for cracked laminates, Int. J. Fracture, 36(2) (1988) 101-119.

[9]Suo Z, Hutchinson J W, Interface crack between two elastic layers, Int. J. Fracture, 43(1) (1990) 1-18.

[10]Schapery R A, Davidson B D, Prediction of Energy Release Rate for Mixed-Mode Delamination Using Classical Plate Theory, Appl. Mech. Rev., 43 (5S) (1990):S281-S287.

[11]Davidson B D, Kruger R, Konig M, Three-dimensional analysis of center-delaminated unidirectional and multidirectional single-leg bending specimens, Compos. Sci. Technol., 54(4) (1995) 385-394.

[12]Armanios E A, New methods of sublaminate analysis for composite structures and applications to fracture processes, Georgia Institute of Technology, Georgia, 1985.

[13]Chatterjee S N, Ramnath V, Modeling laminated composite structures as assemblage of sublaminates, Int. J. Solids Struct., 24 (5) (1988) 439-458.

[14]Li S, Wang J, Thouless M D. The effects of shear on delamination in layered materials, J. Mech. Phys. Solids, 52(1) (2004) 193-214.

[15]Bruno D, Greco F. Mixed mode delamination in plates: A refined approach, Int. J. Solids Struct., 38 (50-51) (2001) 9149-9177.

[16]Wang J, Qiao P, Interface crack between two shear deformable elastic layers, J. Mech. Phys. Solids, 52(4) (2004) 891-905.

[17]Qiao P, Wang J, Mechanics and fracture of crack tip deformable bi-material interface, Int. J. Solids Struct., 41(26) (2004) 7423-7444.

[18]Wang J, Qiao P, On the energy release rate and mode mix of delaminated shear deformable composite plates, Int. J. Solids Struct., 41(9-10) (2004) 2757-2779.

[19]Wang J, Qiao P, Interface crack between two shear deformable elastic layers, J. Mech. Phys. Solids, 52(4) (2004) 891-905. 